\title{
ELECTRON FIELD EMISSION AND THE SPACE CHARGE LIMIT: TECHNIQUES AND TRADEOFFS
}

\author{
Dave Morris $^{*}$ and Brian Gilchrist ${ }^{\dagger}$ \\ University of Michigan, Ann Arbor, MI, 48109
}

\begin{abstract}
$\underline{\text { ABSTRACT }}$
Cold cathode electron emission technology is being developed in a variety of forms for spacecraft charge control applications, ranging from solar panel protection to deep space electric propulsion. Cold cathode emission can provide significant efficiency savings (power, weight, space, etc.) over classic approaches (such as thermionic emitters and plasma contactors) for many classes of missions. However, regardless of the technology approach (closely matched gate-tip arrays, gridded carbon nano-tube plates, or more exotic techniques) for applying an electric field to a novel emitting surface, all are constrained by the space charge limits which apply whenever charge moves (at a certain velocity and density) across a gap (with a certain size and potential). No matter how efficiently the electrons are extracted from the emitter material, at least enough energy must be added to avoid the space charge limit, and this can be a significant additional cost in some applications.
\end{abstract}

Analytic solutions for the space charge limit in simple geometries have been developed up to three dimensions, but there are a variety of factors that can be manipulated in the design of a physical system that cannot be practically and generically included in such analysis. Via particle-in-cell modeling, a number of techniques for mitigating the space charge limit are being studied, ranging from atypical geometries to spatial and chronological distribution of emission. Some results of explorations of these techniques will be presented here.

\section{NOMENCLATURE}

$\mathrm{FE}=$ field emitter

$\mathrm{SCL}=$ space charge limit

$\mathrm{PIC}=$ particle-in-cell

$\mathrm{Vgt}=$ gate tip voltage

$\varepsilon_{0}$ : permittivity of free space

e: electron charge $[\mathrm{C}]$

$\mathrm{m}_{\mathrm{e}}$ : electron mass $[\mathrm{kg}]$

$\mathrm{T}_{\mathrm{o}}$ : electron emission energy $[\mathrm{eV}]$

$\mathrm{D}$ : gap spacing [m]

$\mathrm{V}$ : gap voltage [V]- equal to spacecraft bias with respect to the plasma

$\mathrm{W}$ : emitter width

$\mathrm{r}_{\mathrm{b}}$ : emitter radius

A: emitter area $\left[\mathrm{m}^{2}\right]$

$\mathrm{s}=$ sheath size $[\mathrm{m}]$

$\mathrm{J}_{\mathrm{CL}}(\mathrm{N})=\mathrm{N}$ dimensional Child-Langmuir

current limit $\left[\mathrm{A} / \mathrm{m}^{2}\right]$

\section{INTRODUCTION}

There are a variety of space applications that require electron charge emission. Any electric propulsion thruster (Hall Thrusters, Ion thrusters such as DS-1, etc.) requires the simultaneous emission of electrons to balance the emitted ion charge. Space electrodynamic tethers generate thrust via

\footnotetext{
* Graduate Student, Electrical Engineering and Space Systems

${ }^{\dagger}$ Professor, Electrical Engineering and Space Systems, AIAA Associate Fellow
} 
interaction between a current flowing through the tether and the Earth's (or Jupiter's) magnetic field. This requires the energy efficient emission of electrons at one end of the system. Also, low-power electron emission can be useful for spacecraft charge control applications.

Electron field emission (FE), also known as cold-cathode electron emission, can achieve low power emission with no consumables. FE devicess do so using small-scale structures wherein a relatively small applied voltage (10s to 100 s of volts) creates very large fields $\left(>10^{9} \mathrm{~V} / \mathrm{m}\right)$. The strength of the field at the emitting surface is due to the small distance (micron scale) between the surface and the gate. Electrons are pulled free via quantum tunneling and accelerated through the gate and away from the spacecraft. Using semiconductor fabrication techniques, cold cathode field emitters can be built on large scales at low cost. Some practical issues relating to contamination, atomic oxygen tolerance, and robustness in general, are still being resolved for space applications, but FE devices are already in expanding use in terrestrial applications, and research for space applications is currently underway.

The focus of this paper is mitigation of space charge limits on electron injection via electron field emission. FE devices emit charge with an initial velocity depending on the gate-tip voltage and the voltage of any other associated acceleration or protection grids. As electrons leave the emitter into free space, or a plasma, each electron emitted experiences a deceleration caused by the electric field of the electrons before it. If the emission density is too high, this effect will decelerate the charge and reverse the flow and reflect current back on the emitter and spacecraft. The current level where this first occurs is referred to as the space charge limit (SCL) [Child, 1911], While the scales and quantities of emission presented here are tuned to typical field emitter values, most of the results are equally applicable to thermionic or other low velocity/high density charged beam emission.

\section{FE SYSTEMS}

Figure 1 below shows one type of $\mathrm{FE}$ device, in this case known as a Field Emitter Array (FEA, or sometimes FEAC indicating its cathode functionality). The scale is sub micrometer.

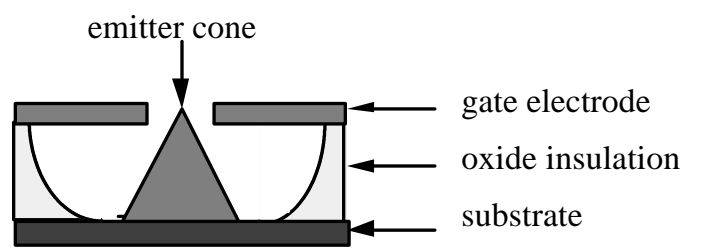

Figure 1: Close in view of a single FEAC tip. A typical array will consist of thousands to millions of tips.

A typical array is a semiconductor wafer with up to millions of individual tips per square centimeter. Typical operating voltages are in the range of 50-100 V, and typical currents can be as high as $1 \mathrm{~mA}$ per tip (though SCLs and other factors prevent all the tips from emitting at this level at once).

Other examples of FE systems include carbon-nanotube emitters, textured surface emitters and more recently, triple point emitters. All of these technologies function in much the same way; a close gate structure generates large fields at the emitting surface with reasonable externally applied voltages. FEACs and some other types of FE devices also make use of field focusing via the geometry of the surface to increase the local electric field strength. Regardless, the emitting surface emits electrons once the surface field is strong enough to effect quantum tunneling, and these electrons are 
accelerated away from the emitter and spacecraft by the same electric field [Jensen, 1999].

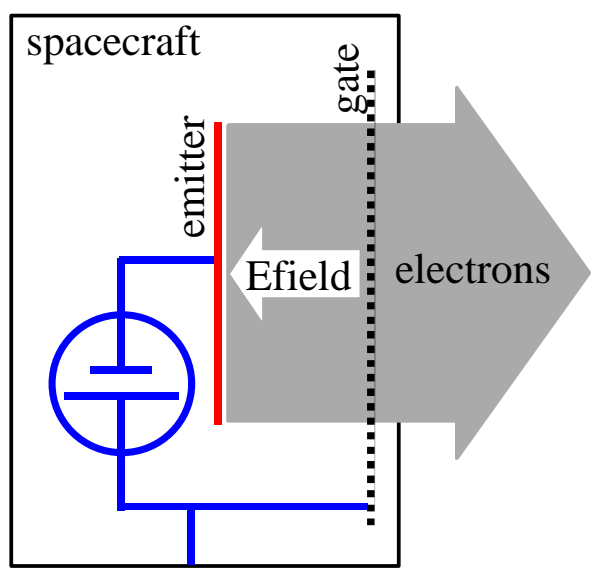

Figure 2: General Field Emitter Structure

The advantages of FE systems over alternative electron emission devices are lower power requirements and no consumables. Hollow cathode contactors, which operate by generating a dense plasma plume and injecting electrons into the ambient plasma across a double layer sheath, use a consumable gas supply and require heater power, but may need the least drive power to inject electrons into the plasma, due to the large surface area. Electron guns (aka thermionic emitters) require significant heater power and higher injection voltages than FE devices because the current is generated in a smaller area and are also sensitive to neutral gas exposure.

With a small increase in FE system complexity, secondary gate structures can be added to adjust a field emitter's final emission velocity. The initial velocity depends on the technology and chemistry of the emitter surface and the field strength it requires. With a second gate, electrons can be accelerated or decelerated to a final velocity barely above the minimum energy required to avoid space charge limits. This conserves energy on it's own (and provides ion protection [Marrese, 1999]).
Additionally, with this capability any other techniques used to improve the space charge limit can also be readily translated into overall power savings.

\section{SPACE CHARGE LIMIT}

Electrons leaving the FE device have a kinetic energy corresponding to the potential that they were accelerated through after extraction from the conductor. In a device operating at $50 \mathrm{~V}$, this corresponds to a velocity of about $4 \times 10^{6} \mathrm{~m} / \mathrm{s}$.

The problem arises when the level of current (number of electrons) emitted exceeds the space charge limit. The electric field created by each emitted electron acts to cancel any anode-to-cathode potential electric field and decelerate new electrons that are emitted. This continues for as long as the electrons are present in the low density sheath surrounding the spacecraft or connecting the electron emission with ion beam in a EP thruster. Once the electrons have been accommodated by the plasma, the objective of the emitter has been achieved and they can be ignored. (Additional analysis and simulations are still underway to verify where exactly this takes place.) However, if sufficient charge is emitted into the sheath the deceleration of electrons can become large enough to reverse the electron flow back to the emitter and spacecraft. This effect is known, in general, as space charge limited flow and was first studied at the beginning of the 20th century by Child and Langmuir (Child, 1911), resulting in the commonly accepted Child-Langmuir law for space charge limited flow given in equation 1.

$$
J_{C L}(1)=\frac{4 \varepsilon_{0}}{9 D^{2}} \sqrt{\frac{2 e}{m}} V^{\frac{3}{2}}
$$

Equation 1 describes the current density that will flow from one infinite plane to another 
based on the voltage between the planes $(\mathrm{V})$ and the distance between them (D). This was originally solved for vacuum tube technology, where one plane is a thermionic emitter boiling off an effectively infinite supply of electrons at thermal velocities (essentially zero compared to the voltages involved), and the other plane is a collector biased to some positive voltage.

This equation has been expanded to cover the situation where the electrons have an initial velocity, as they do when they leave a field emitter. In one dimension and with an initial emission velocity assumed, the spacecharge current limited condition is given in the equation below. (Luginsland, 1998)

$$
I_{C L}(1)=\frac{4 \varepsilon_{o}}{9 e} \sqrt{\frac{2}{m_{e}}} \frac{T_{o}^{3 / 2}}{D^{2}}\left[1+\sqrt{1+\frac{e V}{T_{o}}}\right]^{3} A
$$

When the above current is exceeded, a portion of the emitted current returns to the spacecraft, limiting its effectiveness.

To illustrate space charge limitations the following figures show results of one dimensional particle-in-cell (PIC) simulation in the space charge limited regime. XPDP1 [Verboncoeur et al., 1993] simulates particle activity between two plates with each particle representing an infinite sheet of charge (thus the 1D nature). The first plot (Figure 3 ) is of velocity versus position for the individual particles in the simulation (the apparently continuous line is actually individual simulation particles in close proximity). The center of the vertical axis is zero velocity. Electrons are emitted with $60 \mathrm{eV}$, but the space charge effect decelerates and actually reverses the direction of some. The situation shown in Figure 3 below is not a steady state solution but rather one instant in time.

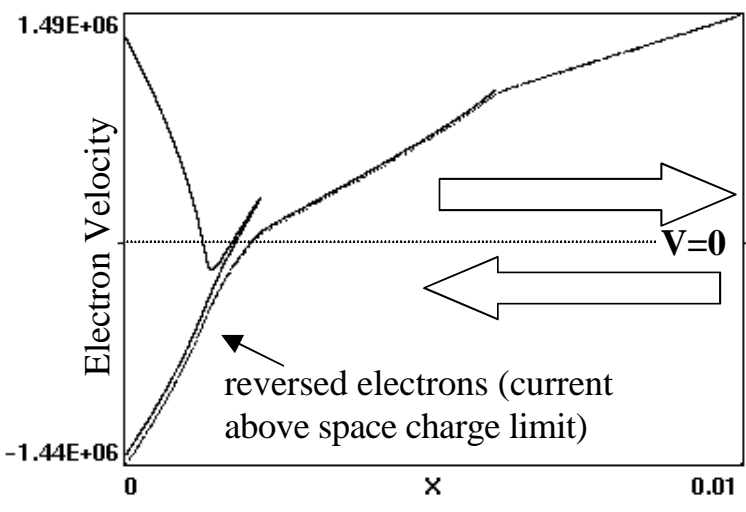

Figure 3: 1 dimensional charge emission, with buildup and reflection near the emission point (left side).

Figure 4 shows a plot of current collected at the anode (right side) versus time. The oscillatory nature reflects the process of electron bunching and spreading in between the plates. This bunching of electrons is referred to as a virtual cathode, and the timing of this process will become important later on.

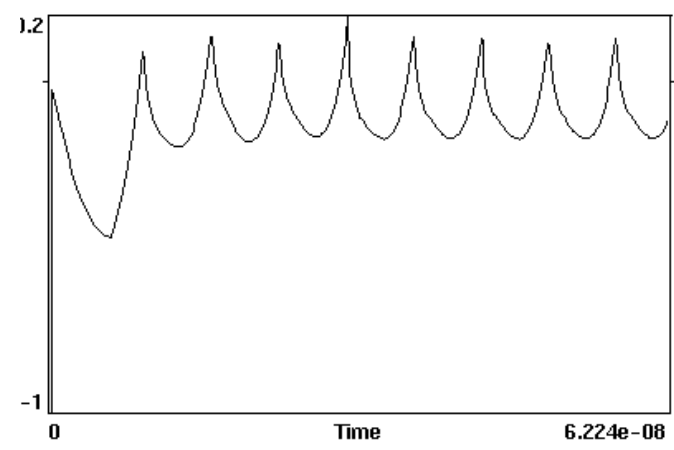

Figure 4: emitted current in the space charge limited regime.

These figures specifically are for 1A current emission across a $1 \mathrm{~cm} 1 \mathrm{~V}$ sheath. This simulation in general represents the approximation where the sheath is considered to be the only limiting factor- i.e. any electrons managing to cross the sheath are absorbed by a virtual anode (the ionosphere) and need no longer be considered. Once across the sheath, the low energy ambient electrons are displaced and the slowly moving ambient ions help balance the incoming electrons, thus 
reducing the net charge imbalance (Gilchrist, 1999). As an illustration, the following diagram shows how the emitter would interact with the sheath and the ionosphere during a tether propulsion mission.

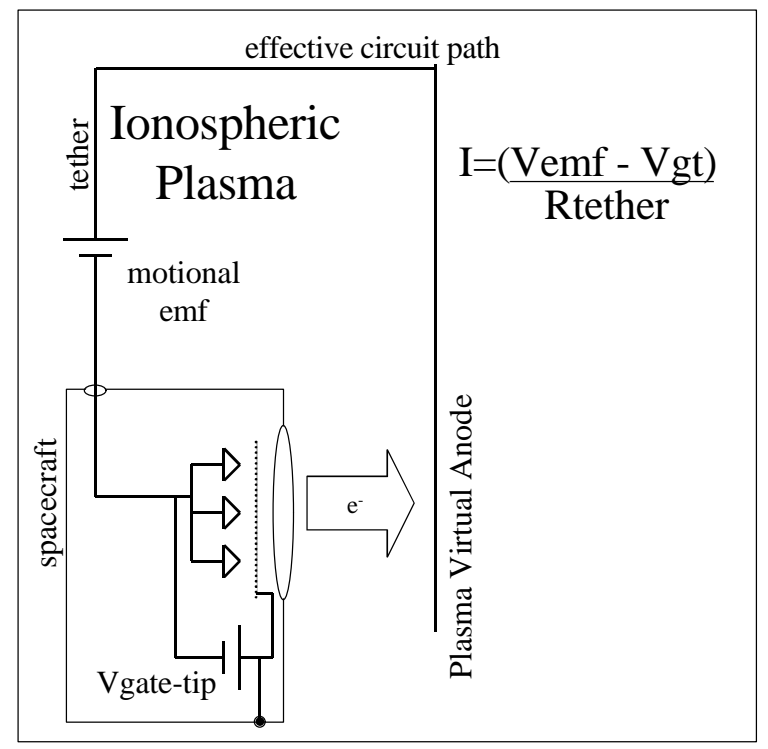

Figure 5: System diagram of an electrodynamic tether propulsion mission. The emitter's task is to move electrons away from the spacecraft into the surrounding plasma.

\section{$\underline{\text { Sheath Size and Spacecraft Charging }}$}

As equation 2 indicates, the space charge limit increases with the gap voltage that the electrons are emitted across. Simultaneously, however, the current limit decreases as the gap size increases. For ionospheric applications it is assumed that the relevant distance is the width of the sheath around the spacecraft. This sheath increases in size with the voltage difference between the spacecraft and the plasma. Therefore there are counterbalancing effects as the spacecraft biases negative with respect to the plasma. The gap voltage increases, but so does the gap size. The relationship between the size of the sheath and the potential of the spacecraft for small one-dimensional sheaths is given the following equation [Lieberman \& Lichtenberg, 1994].

$$
s=\left(\frac{2 \varepsilon_{0} V}{e n_{s}}\right)^{1 / 2}
$$

This works out to $1.5 \mathrm{~cm}$ for a spacecraft at $-1 \mathrm{~V}$ floating potential relative to a $5 \times 10^{5} / \mathrm{cm}^{3}$ plasma. Plugging this into equation 2 it can be shown (see plot below) that for this sheath model increasing the spacecraft bias reduces the space charge current limit. The detrimental effect of having to traverse a greater distance is stronger than the beneficial effect of a greater bias across the gap. The best situation therefore is to keep the spacecraft potential low and the resulting sheath dimension small. In the low earth orbit environment a typical spacecraft floating potential (unaffected by tethers or ion emission or solar panel charging) is about -1 $\mathrm{V}$. Therefore in this paper it is assumed that the goal of the FE system is to keep the spacecraft as near this potential as possible.

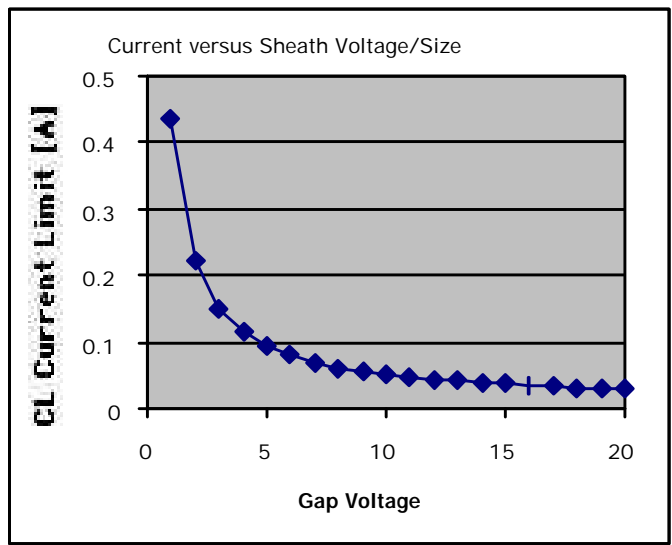

Figure 6: The coupled effect on the space charge limit of gap size (sheath size) and gap voltage (spacecraft bias)

In the most simple one dimensional analysis, with a $-1 \mathrm{~V}$ spacecraft bias, a $50 \mathrm{eV}$ electron emission, and a $1.5 \mathrm{~cm}$ gap, the space charge current limit is $3 \mathrm{~mA} / \mathrm{cm}^{2}$ (based on Equation 1). This is an effective lower limit to the 
ability of an FE to emit charge (assuming that crossing the sheath is the primary limitation). $1 \mathrm{~A}$ of emission would therefore require a minimum of just over $300 \mathrm{~cm}^{2}$ of emitting area. The goal of the rest of the paper is to improve on this result.

\section{$\underline{\text { Beam Spreading and Higher Dimensions }}$}

More detailed analysis indicates that significant improvement can be attained by considering spreading of the emitted electrons in multiple dimensions. Luginsland [1996] suggests that the improvement of going to a long, thin emitter (modeled as an infinite strip) would be a factor of 2 (using a $0.5 \mathrm{~cm}$ wide emitter, $1.5 \mathrm{~cm}$ sheath). This result, given in equation 4, was derived via numerical analysis of computer simulation results. This would reduce the required area to $150 \mathrm{~cm}^{2}$ for 1 amp at $50 \mathrm{~V}$ gate-tip.

$$
\frac{J_{c l}(2)}{J_{c l}(1)}=1+\frac{0.3145}{W / D}-\frac{0.0004}{(W / D)^{2}}
$$

This equation was expanded to three dimensions by Lau [2001] shown in equation 6 , with $\mathrm{D}$ being the distance from the emitter to the anode, $\mathrm{W}$ being the width of the anode, and $\mathrm{R}$ being the radius (an elliptical emitter is thus assumed).

$$
\frac{J_{C L}(3)}{J_{C L}(1)} \approx 1+\frac{D}{\pi W}+\left(\frac{1}{4}-\frac{1}{2 \pi}\right) \frac{D}{R}
$$

Equation 6 works well for situations where $\mathrm{R} / \mathrm{D} \gg>1$, but it has the limitation that it does not consider beam spreading. With emitters that are small compared to the gap size $(\mathrm{D}>=\mathrm{R})$ further improvement is obtained by considering three-dimensional spreading of the beam. A theoretical calculation by Humphries [1990] suggests an improvement of 2.5 for a $1 \mathrm{~cm}^{2}$ $\left(\mathrm{r}_{\mathrm{b}}=0.564 \mathrm{~cm}\right), 1.5 \mathrm{~cm}$ sheath case, as per equation 8 .

$$
\frac{J_{C L}(3)}{J_{C L}(1)}=\frac{\left[r_{b}^{2}+(D / 2)^{2}\right]}{r_{b}^{2}}=\left[1+\left(D / 2 r_{b}\right)^{2}\right]
$$

These equations can be easily compared using particle in cell simulation codes. Analysis was done in XOOPIC [Verboncoeur, 1993], which is a 2.5D code (i.e. tracking 2 physical dimensions and 3 velocity dimensions). The following figure shows the setup for the XOOPIC simulations. The simulation uses radial symmetry along the axis of the emitted beam.

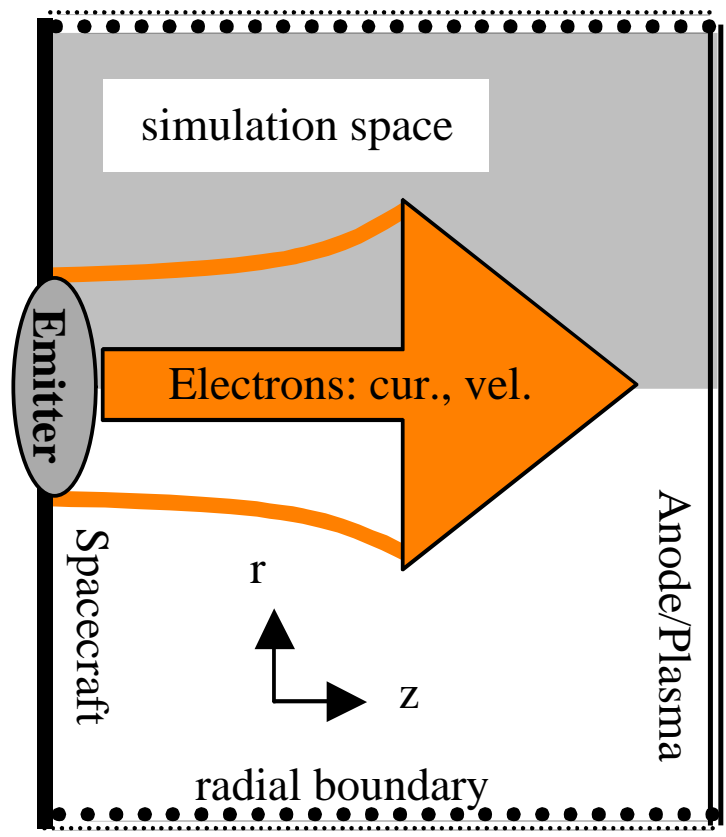

Figure 7: Setup of the simulation space in XOOPIC. The simulation is $2.5 \mathrm{D}, 2$ dimensions in space, 3 dimensions in velocity.

The next figure shows a summary of the higher dimension beam spreading theories and their range of agreement with simulations done in XOOPIC. The SCL is plotted versus the size of the emitter. At large sizes $(10 \mathrm{~cm}$ wide emitter- much larger than the $1 \mathrm{~cm}$ gap used in this simulation), the geometry is similar to an infinite plane, 
and the 1D analysis is sufficient. It is only when the emitter size drops to about the same as or smaller than the gap size that a three dimensional analysis is important. At still lower emitter sizes, the inclusion of beam spreading becomes critical. At the smallest sizes, only the Humphries equation agrees with the simulated results. Improvement of several hundred times over the 1D SCL is possible in extreme cases (mm or smaller emitter sizes).

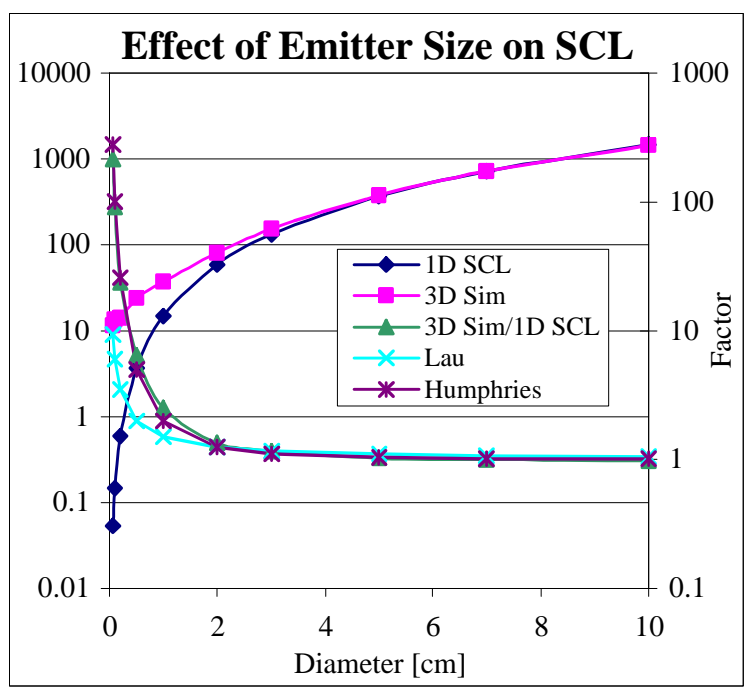

Figure 8 : Theoretical 1D and simulated 3D SCL, for 100V emission across a $1 \mathrm{~cm} 1 \mathrm{~V}$ gap. Diamond and square lines indicate SCLs with the scale to the left. Star and asterisk lines indicate the theoretically calculated improvement factor over 1D and the triangle line is the simulated improvement factor all measured on the scale to the right.

The important conclusion of these results is that beam spreading from small emitters allows a vast improvement in the space charge limit over wide emitters. For a spacecraft where emission power is the most critical feature, the best solution may be a number of button sized emitters spread around the spacecraft. The space between these emitters must be sufficiently large that their respective beams do not overlap enough for the combined density to exceed the SCL. As one example, figure 7 shows beam spreading for 81 volt emission from a $1 \mathrm{~cm}$ diameter emitter versus gap size. The spreading is plotted on the left normalized to the size of the emitter. There is a strong relationship between gap size and emitter spacing- which will be especially critical on spacecraft emitting charge across a large sheath.

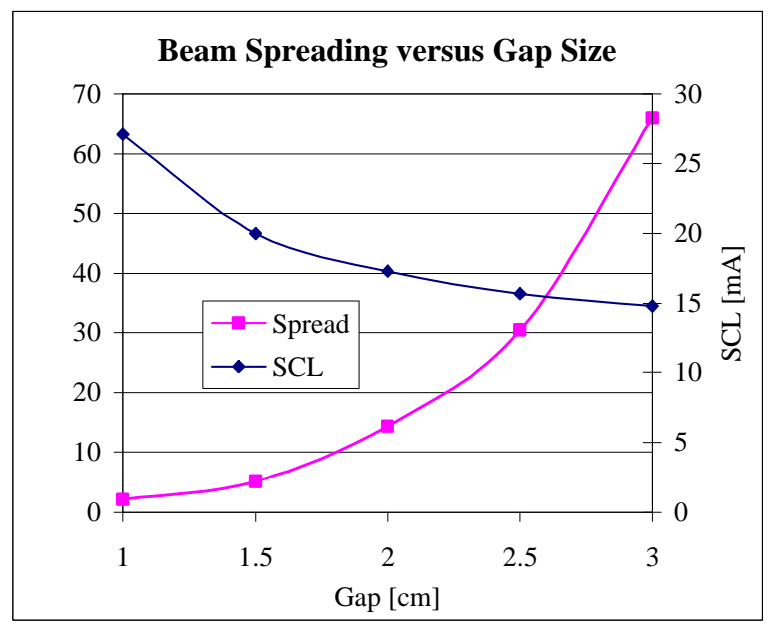

Figure 9: Beam spreading across a $1 \mathrm{~cm}$ gap

If higher current levels are required, additional techniques can be implemented to further capitalize on beam spreading. Natural self-field spreading can be improved upon by installing electron optics (in the form of curved acceleration grids or rings) to further spread the beam starting closer to the emitter.

\section{Frequency Effects}

In addition to spatial spreading of the emitted beam, temporal spreading can be used to help mitigate the space charge limit. Figure 4 shows the oscillatory nature of current emitted at a level above the space charge limit. The physics behind this are that the charge decelerates, accumulates with the emitted charge behind it (forming a virtual cathode), and then disperses from self-field forces. The virtual cathode is transient, and disperses on a time scale related to the beam velocity and density. This phenomenon can be taken advantage of by pulsing the emitted beam near the same frequency, so that as the virtual cathode begins forming the emitted beam density decreases preventing the 
virtual cathode from reaching critical mass. [Winglee, 1987] The beam density remains low long enough for the virtual cathode to dissipate without returning charge to the spacecraft. Once it is dissipated the emitted beam can ramp back up to higher densities again. The following figure shows the peak current possible for a $10 \mathrm{~cm}$ diameter emitter operating across a $1 \mathrm{~cm}$ gap, with sinusoidal emission modulation of the emitted beam.

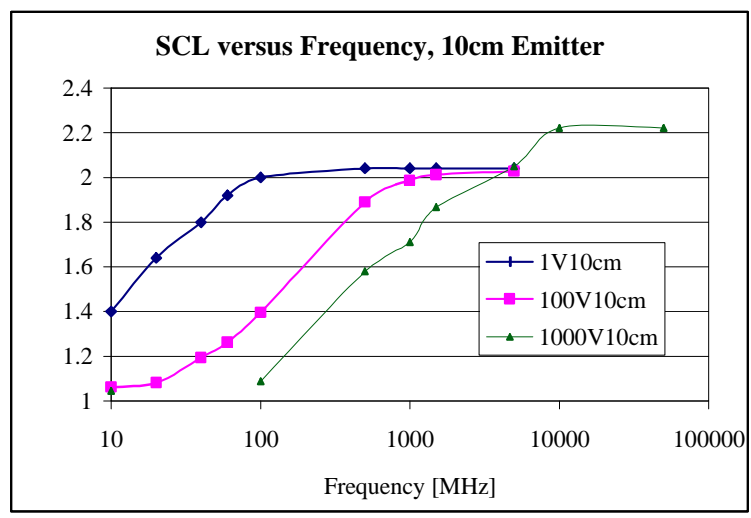

Figure 10: The effect of frequency modulation of emission current on the space charge limit for three emission velocities. The gap is $1 \mathrm{~cm}$.

Just the magnitude (number of electrons, or beam density) of the current is modulated, not the velocity (in a physical FE, these variables would be linked). The average density of a beam with a sinusoidal variation is half of the maximum amplitude. As the plot shows, once the frequency reaches a sufficiently high value the SCL becomes twice what it is in the DC case (the DC case is normalized to 1 ). But this is no gain because the average current at the high frequency SCL is the exact same level as with DC emission. It is possible that at some combination of velocities and frequencies, perhaps at much higher emission velocities where the beam plasma frequency and the required emission modulation frequency become similar, that the modulated emission will on average exceed the DC value, but additional analysis will be required.
Even with no gain for an individual emitter, there is a possibility of gain via emission modulation when you have multiple emitters operating in near proximity but out of phase. The following figure shows what this would look like.

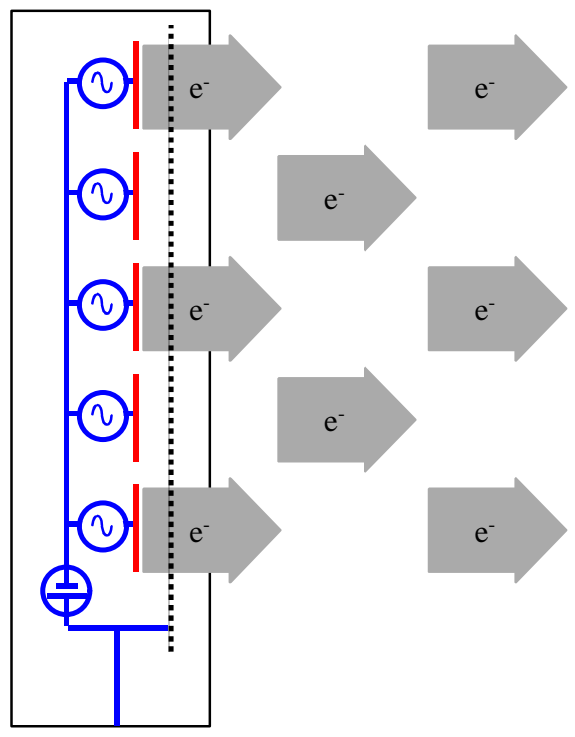

Figure 11: Proximate emitters where time phasing isolates emitters temporally to prevent adjacent emitters from compounding space charge limits

In this scenario the overall emission area is minimized (emitters are in close proximity) but temporal isolation reduces the overlap of emitted beams. The packets of charge are able to expand more than with continuous emission, possibly improving SCLs. The gain of such a system has yet to be proven, and it would have to be significant to justify the additional complexity. The likely application is a mission where spacecraft surface area is at a premium.

\section{SUMMARY}

Electron field emitter systems are becoming an enabling technology for a variety of space missions in the near future. In order to optimize efficiency for the inevitable limiting factors of power, area, mass, and cost, considerations of geometry and temporal and spatial modulation to mitigate 
the space charge limits will need to be made. The techniques and results presented here are a beginning to these analysis.

Ongoing research includes additional work with spatial and temporal modulation, research into defocusing structures, and research into physical and electrical integration issues for development of practical space electron field emission systems.

\section{$\underline{\text { Acknowledgement }}$}

This work is being funded by a NASAMSFC GSRP student grant.

\section{$\underline{\text { References }}$}

Child, C. D., "Discharge from Hot CaO", Phys. Rev., ser. 1, vol. 32, 1911

K. L. Jensen, Physics of Plasmas 6, 2241, (1999).

Gilchrist B. K., Jensen, A. Gallimore, J. Sorensa, Field-Emitter Array Cathodes (FEACs) for Space-Based Applications: An Enabling Technology, white paper, The University of Michigan and The Naval Research Laboratory, 1999

Lau, Y. Y., "A simple Theory on the Two-Dimensional Child-Langmuir Law", Phys. Rev. Lett. 87, 278301, 2001.

Lieberman, Lichtenberg, Principles of Plasma Discharges and Materials Processing, John Wiley \& Sons, Inc., 1994

Luginsland, J., S. McGee, and Y. Y. Lau, IEEE Trans. Plasma Sci., 26, p.p. 901-904, 1998.

Luginsland, J. W., Y. Y. Lau, R. M. Gilgenbach, Two-dimensional Child-Langmuir law, Phys. Rev. Lett., 77, p. 4668, 1996.

Marrese, C., Compatibility of field emission cathode and electric propulsion technologies: theoretical and experimental performance evaluations and cathode requirements, Ph.D., University of Michigan, 1999.

NRL Plasma Formulary published by the Naval Research Laboratory, 1998 edition. NRL/PU/6790--98-358

Verboncoeur, J. P., M. V. Alzes, V. Vahedi, C. Birdsall, Simultaneous Potential and Circuit Solution for 1-D Bounded Plasma Particle Simulator Codes, J. Comp. Phys., V. 104, p321-328, 1993

Winglee, R.M., P.L. Pritchett, "Space Charge Effects During the Injection of Dense Electron Beams Into Space Plasmas", Journal of Geophysical Research, Vol. 92, No. A6, Pages 6114-6126, 1987 